\title{
Finite-state phrase parsing by rule sequences
}

\author{
Marc Vilain and David Day \\ The MITRE Corporation \\ 202 Burlington Rd. \\ Bedford, MA 01720 USA \\ mbv@mitre.org,day@mitre.org
}

\begin{abstract}
We present a novel approach to parsing phrase grammars based on Eric Brill's notion of rule sequences. The basic framework we describe has somewhat less power than a finite-state machinc, and yet achieves high accuracy on standard phrase parsing tasks. The rule language is simple, which makes it easy to write rules. Further, this simplicity cnables the automatic acquisition of phraseparsing rules through an error-reduction strategy.
\end{abstract}

This paper explores an approach to syntactic analysis that is unconventional in several respects. To begin with, we are concerned not so much with the tradi tional goal of analyzing the comprehensive structure of complete sentences, as much as with assigning partial structure to parts of sentences. The fragment of interest here is demonstrably a subset of the regular scts, and while these languages are traditionally analyzed with finitc-state automata, our approach rclics instcad on the rule sequence architecture defined by Eric Brill.

Why restrict ourselves to the finite-state case? Some linguistic phenomena are easier to model with regular sets than context-free grammars. Proper names are a case in point, since their syntactic distribution partially overlaps that of noun phrases in general; as this overlap is only partial, name analysis within a full context-free grammar is cumbersome, and some approaches have taken to include finite-state name parsers as a front-end to a principal context-free parsing stage (Jacobs et al. 1991). Proper names are of further interest, since their identification is independently motivated as valuable to both information retrieval and extraction (Sundheim 1996). Further, several promising recent approaches to information extraction rely on little more than finitestate machines to perform the entire extraction analysis (Appelt et al. 1993, Grishman 1995).

Why approach this problem with rulc sequences? In this paper we maka the case that rule sequences succeed at this task through their simplicity and speed. Most important, they support mixed-mode acquisition: the rules are both easy for an engineer to write and easy to lcarn automatically.

\section{Rule sequences}

As part of our work in information extraction, we have been extensively exploring the use of rule sequences. Our information extraction prototype, Alembic, is in fact based on a pipcline of rule sequence processors that run the gamut from part-of-speech tagging, to phrase identification, to sentence parsing, to inference (Aberdecen et al. 1995). In cach case, the underlying method is identical. Processing takes place by sequentially relabeling the corpus under consideration. Each scquential step is driven by a rule that attempts to patch residual errors left in place in the preceding steps. The patching process as a whole is itself preceded by an initial labeling phase that provides an approximate labeling as a starting point for rule application.

This patching architecture, illustrated in Fig. 1, was codified by Eric Brill, who first exploited it for part-ofspeech tagging (Brill 1993). In the part-of-speech application, initial labeling is provided by lexicon lookup: lexemes are initially tagged with the most common part of speech assigned to them in a training corpus. 'This initial labeling is refined by two sets of transformations. Morphological transformations relabel the initial (default) tagging of those words that failed to be found in the lexicon. The morphological rules are followed by contextual transformations: these rules inspect lexical context to relabel lexemes that are ambiguous with respect to part-of-specch. In cffect, the morphological transformations patch crrors that were due to gaps in the lexicon, and the contextual rules patch errors that were due to the initial assignment of a lexeme's most common tag.

\section{Phrase identification: some examples}

Sequencing, patching, and simplicity, the hallmarks of Brill's part-of-specch tagger, are also characteristic of our phrase parser. In our approach, phrases are initially built around word sequences that meet certain lexical or part-of-specch criteria. The sequenced phrase-finding rules then grow the boundaries of phrases or set their label, according to a repertory of simple lexical and contextual tests. For example, the following rule assigns a label of ORG to an unlabeled phrase just in case the phrase is ended by the word "Inc."

\begin{tabular}{|c|c|c|}
\hline \multicolumn{3}{|l|}{ (def-phraser } \\
\hline label & NONE & $\begin{array}{l}\text { : phrase is currently } \\
\text {; unlabelled }\end{array}$ \\
\hline right-wd-1 & lexeme "Inc." & $\begin{array}{l}\text { : rightmost word in the } \\
\text { : phrase is "inc." }\end{array}$ \\
\hline label-action & $O R G)$ & $\begin{array}{l}\text {; change the phrase's label, } \\
\text { : but not its boundaries }\end{array}$ \\
\hline
\end{tabular}

Now, consider the following partially labelled string:

$<$ none $>$ Donald F. DeScenza</nones, analyst with

$<$ none $>$ Nomura Securities Inc.</nones 


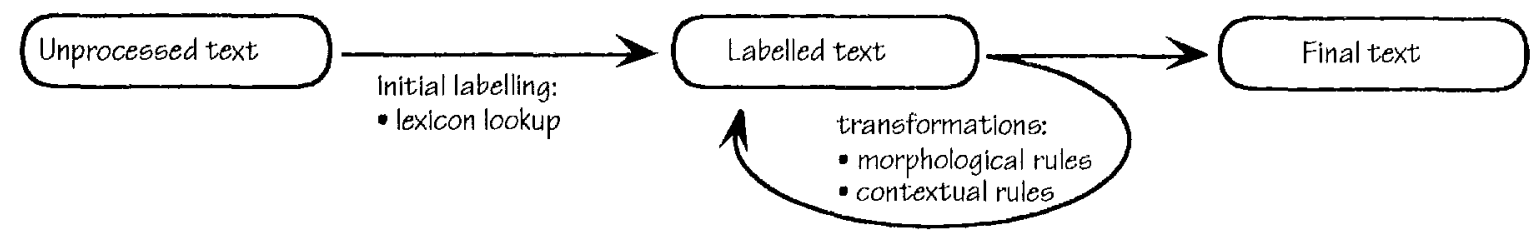

Figure 1: Brill's rule sequence architecture as applied to part-of-speech tagging.

The sGmL markup delimits phrases whose boundaries were identified by the initial phrase-finding pass. Of these phrases, the sccond successfully triggers the example rule, yielding the following relabeled string.

$<$ none $>$ Donald F. DeScenza</none>, analyst with $<$ org $>$ Nomura Securities Inc.<lorg>

'The rule, which seems both as obvious as walking and as fool-proof comes from the name-finding processor we developed for our participation in the $6^{\text {th }}$ Message Understanding Conference (MUC-6). As it turns out, though, the rule is in fact not crror-proof, and causes both crrors of omission (i.e. recall errors) and commission (i.e. precision errors). Consider the case of "Volkswagen of America Inc." Because the initial phrase labeling is only approximate, the string is broken into two sub-phrases scparated by "of".

<none> Volkswagen</none> of <none>America

Inc. $<$ /nones

The example rule designates the partial phrase "America Inc." as an ORG, a precision error because of its partiality, and fails to produce an ORG label spanting the cutire string (a recall error).

<none $>$ Volkswagen</nones of <org $>$ America Inc.<lorg $>$

This problem is patched by a subsequent namefinding rule, namely the following.

\begin{tabular}{|c|c|c|}
\hline ef-phraser & & \\
\hline label & ORG & ; this is an organization \\
\hline left-wd-1 & test country? & $\begin{array}{l}\text {; is the leftmost lexeme } \\
\text {; in the phrase on a list } \\
\text {; of country words? }\end{array}$ \\
\hline left-ct $\times t-1$ & lexeme "op" & $\begin{array}{l}\text {; to the left of the } \\
\text {; phrase is the word "op" }\end{array}$ \\
\hline left-ctxt-2 & phrase NONE & $\begin{array}{l}\text { : to the left of that is an } \\
\text {; unlabelled phrase }\end{array}$ \\
\hline bounds-actior & MERGE & ; merge the entire left \\
\hline labbel-action & ORG) & $\begin{array}{l}\text { : contextinto the ORG, } \\
\text {; phrase and all }\end{array}$ \\
\hline
\end{tabular}

The first two clauses of the rule are antecedents that look for phrascs such as "America Inc." 'l'he next two clauses are further antecedents that look to the left of the phrase for contextual patterns of form

"<none $>$... $<$ /none of".

'The final two clauses incorporate the left context wholesale into the triggering phrase, yielding:

<org>Volkswagen of America Inc.<lorg>

This rule effectively patches the errors caused by its predecessor in the rule sequence, and simultancously climinates both a recall and a precision crror.

\section{The phrase finder}

With these cxamples as background, we may now turn our attention to the technical details of the phrase finding process. As noted above, this process occurs in two main steps, an initial labcling pass followed by the application of a rule sequence.

\section{Initial phrase labeling}

'The initial labeling process seeds the phrase-finder with candidate phrases. 'Ihese candidate phrases need not be any more than approximations. In particular, it is not necessary for these candidates to have wholly accurate boundaries, as their left and right edges can be adjusted later by means of patching rules. It is also not necessary for these candidates to be unfragmented, as fragments can be reassembled later, just as with "Volks wagen of America Inc." Further, applications that require multiple types of phrase labels, necd not choose such a label during the initial phrase-finding pass.

What is important is that the initial phrase identification find the cores of phrases reliably, even if complete phrases are not identified. That is, it must partially align some kind of candidate phrase $x$ for every phrase $\phi$ that is actually present in the input. Extending a concept from information retricval, this amounts to maximizing what we might call initial recall, i.e.,

$\mathrm{R}_{\mathrm{I}}=\left|\Phi_{\mathrm{I}}\right| /|\Phi|$,

where $\Phi$ is the set of actual phrases in a test set, $K$ is the set of candidatc phrases generated by the initial phrasing passs, and $\Phi_{I}$ is the set of those $\phi \in \Phi$ that are partially aligned with some $\kappa \in K$.

The general strategy we have adopted for finding initial phrase seeds is to look for either runs of lexcmes in a fixed word list or runs of lexemes that have been tagged a certain way by our part-of-speech tagger. Differcnt instantiations of this general strategy for initial phrase labeling naturally arise for different phrase-finding tasks. For example, on the classic "proper names" task in mixed-case text, we have achicved good results starting from runs of lexemes tagged with NNP or NNPs, the l'cnn T'reebank proper noun tags. This strategy achieves the desired high initial recall $\mathrm{R}_{\mathrm{I}}$, as these tags are well-correlated with bona fide proper names and are reliably produced in mixed-case text by our part-of-speech tagger. 'I'his strategy docs not yicld quite as good initial precision (i.e., it yiclds false positives) for a number of reasons, such as the fragmentation problems noted above, e.\&.,

\section{Volkswagen/NNP of/N America/NNP Inc./NNF}

Once again, though, these initial precision errors are readily addressed by patching rules. 


\begin{tabular}{|lll|}
\hline Clause type & Syntax & Definition \\
\hline Contextual tests & left-ctxt-1, left-ctxt-2 & Test one place (resp. two places) to the left of the phrase \\
& right-ctxt-1, right-ctxt-2 & Test one place (resp. two places) to the right of the phrase \\
Phrase-internal & left-wd-1, left-wd-2 & Test first (resp. second) word of phrase \\
tests & right-wd-1, right-wd-2 & Test last (resp. next-to-last) word of phrase \\
& wd-ary & Test each word of phrase in succession. Succeeds if any word in the \\
& phrase passes the test. \\
& label-span & Test entire string spanned by phrase \\
& label-action & Test phrase's label \\
Labeltest & Sets the label of the phrase \\
Actions & Modify the phrase's left or right boundaries \\
& & Table l: Repertory of unary rulc clauses. \\
\hline
\end{tabular}

\section{Phrase-finding rules}

A phrase-finding rule in our framework is made up of several clauses. The core of the rulc consists of clauses that test the lexical context around a candidate phrase $\kappa$ or that test lexemes spanned by $\kappa$. 'I'he repertory of these test loci is given in. 'Table 1. At any given locus, a test may either seatch for a particular lexcme, match a lexeme against a closed word list, match a part of speech, or match a phrase of a given type. Most rules also test the label of the candidate phrase $\kappa$.

The unary contextual tests in the table may also be combined to form binary or ternary tests. For example, combining I.ERT-CTXT-I and IEFT-CTXT-2 clauses yields a rule that tests for the left bigram context. This was done in the ORG defragmentation rule described carlier.

A rule also contains at least onc action clause, either a clause that sets the label of the phrasc, or one that modifies the boundarics of the phrase. Finally, some rule actions actually introduce new phrases that embed the candidate and its test context; this allows one to build non-recursive parse trees.

\section{Phrase rule interpreter}

The phrase rule interpreter implements the rule language in a straightforward way. Given a document to be analyzed, it proceeds through a rule sequence one rule $r$ at a time, and attempts to apply $r$ to every phrase in every sentence in the document. "The interpreter first attempts to match the test label of $r$ to the label of the candidate phrase. If this test succeeds, then the interpreter attempts to satisfy the rule's contextual tests in the context of the candidate. If these test succeed, then the rule's bounds and label actions are executed.

Beyond this, the only real complexity arises with phrasc-finding tasks that require one to maintain a temporary lexicon. 'The clearest such example is proper name identification. Indeed, short name forms (e.g., "Detroit Diesel") can sometimes only be identificd correctly once their component terms have been found as part of the complete name (e.g., "Detroit Dicsel Corp."). The converse is also true, as short forms of person names (e.g., "Mr. Olatunji") can help identify full name forms (e.g., "Babatunde Olatunji").
The interpreter maintains a temporary lexicon on a document-by-document basis. livery time the interpreter changes the label of a phrase $\phi$, pairs of form $\langle\lambda, \tau\rangle$ are added to the lexicon, where $\lambda$ is a lexcme in $\phi$, and $\tau$ is the label with which $\phi$ is tagged. This lexicon is then exploited to form the associations betwecn short and long proper name forms (through an cxtension to the rulc repertory defined above).

\section{Correspondence to the regular sets}

It is straightforward to prove that this approach recognizcs a subset of the regular sets, so we will only sketch the ourline of such a proof here. 'l'he proof procecds inductively by constructing a finite state machinc $\mu$ that accepts exactly those strings which reccive a certain label in the phrasc-finding process under a given rulc sequence $\Sigma$. We consider each rule $\rho$ in $\Sigma$ in order, and correspondingly claborate the machine so as to reproduce the rule's effect.

'lo begin with, consider that the initial phrase labeling proceeds by building phrases around lexemes $\alpha_{1}, \ldots, \alpha_{n}$ in a designated word list or by finding runs of certain parts of speech $\pi_{1}, \ldots, \pi_{\mathrm{m}}$. The machine that reproduces this initial labeling is thus

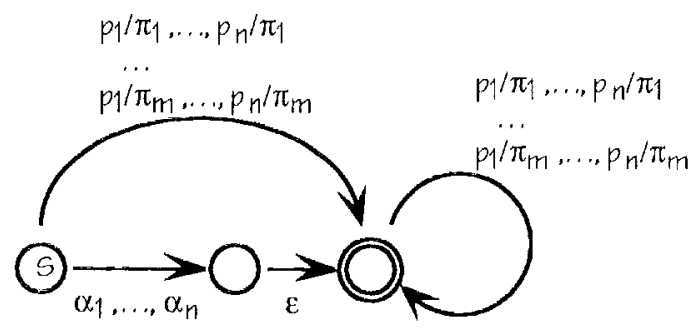

As usual, node labeled " $S$ " is the start state, and any node drawn with two circles is an accepting state. The $\mathrm{p}_{\mathrm{i}} / \pi_{\mathrm{j}}$ arc labels stand for all lexemes in the lexicon that may be labeled with the part of speech $\pi_{j}$.

The induction step in the construction proceeds from $\mu_{i-1}$, the machine built to reproduce $\Sigma$ up through rule $\rho_{i-1}$ in the sequence, and adds additional states and arcs so as to reproduce $\Sigma$ up through rule $\rho_{\mathrm{j}}$.

For example, say $\rho_{\mathrm{i}}$ tests for the presence of a lexeme $\lambda$ to the left of a phrase and extends the phrase's boundaries to include $\lambda$. We extend the machine $\mu$ to 
cncode this rule by replacing $\mu$ 's current start state $S$ with a new one $S$ ', and adding a $\lambda$ transition from $S$ ' to the former start state $S$. Thus

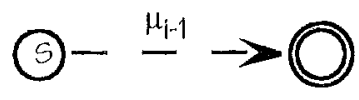

becotnes

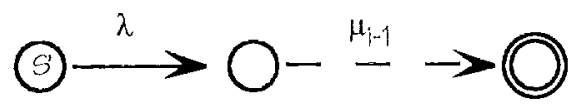

For a rule $\rho_{i}$ that tests whether a phrase contains a certain lexeme $\lambda_{i}$, we construct an "acceptor" machine that accepts any string with $\lambda_{i}$ in its midst.

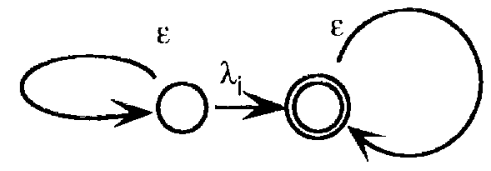

Noting that the regular scts are closed under intersection, we them proceed to build the machine that "intersects" the acceptor with $\mu_{i}$.

Other rule patterns arc handled with constructions of a similar flavor-space considerations preclude their description here. Note, however, that extending the framework with a temporary kxicon makes it transfinite-state, Hinally, as with all semi-parsers, the machines we construct in this way must actually be interpreted as transducers, not just acceptors.

\section{Learning rule sequences automatically}

Our experience with writing rule sequences by hand in this approach has becn very positive. 'Ihe rulc patterns themselves are simple, and the fact that they are sequenced localizes their effects and reduces the scope of their interactions. These hand-engineering advantages are also conferred upot. learning programs that attempt to acquire these rules automatically.

The approach we have taken towards discovering phrase rule secuences automatically is a maximum ceror-rcduction scheme for sclecting the next rule in a sequence. 'This approach originated with Brill's work on part-of-specch tagging and brackcting (Brill 1993).

\section{Brill's rule learning algorithm}

'The scarch for a rulc sequence in a given training corpus begins by first applying the initial labeling function, just as would be the casc in running a complete sequence. lollowing this, the learning procecture needs to consider every rule that can possibly apply at this juncture, which itself is a function of the rule schema language. Lor each such applicable rule $r$ the learner considers the possible improvement in phrase labeling conferred by $r$ in the current state. 'The rule that most reduces the residual error in the training data is selected as the next rule in the sequence.

'This generate-and-test cycle is continued until a stopping criterion is reached, which is usually taken as the point where performance improvement falls below a threshold, or ceases altogether. Other alternatives include setting a strict limit on the number of rules learned, or cross-testing the performance improvement of a rule on a corpus distinct from the training set.

\section{The rule search space}

The language of phrase rules supports a large number of possible rules that the phrase rule learner might need to consider at any one time. 'lake onc of our smaller training sets, in which there are 29I sentences consisting of 6,812 word tokens, with 2,077 unique word types. Considering only lexical rules (those that look for particular words), this means that there are as many as 18,693 possible unary lexical rules $(2,077 \times 9$ rule schemata), and $12,94 \mathrm{I}, 787$ binary lcxical rulcs $\left(2,077^{2} \mathrm{x}\right.$ 3 simple bigram rule schemata) in the search space. However, by inverting the process, and tabulating only those lexical contexts that actually appear in the training texts, this search space is reduced to 2,219 unary lexical rules and 854 binary lexical rules.

There are two substantively different kinds of rules to acquire: rules that only change the label of a phrase, and those that change the boundary of a phrase. 'l he latter present a problem for accurately estimating the improvement of a rule, since sometimes the boundary realignment necessary to fix a phrase problem exceeds the amount by which a single rulc can move a boundary-namely, two lexemes. lior these phrases to be fixed there will have to be more than one rule to nudge the appropriate phrase boundaries over. Wc handle this through a heuristic scoring function that estimates the valuc of moving a boundary in such cascs.

\section{Error estimation methods}

A rule that fixes a problem in some cases might well introduce errors in some other cases. This kind of overgencralization can occur early in the lcarning process, as new rules need only improve over an approximate initial labeling. "The cxtent to which a candidate rulc is rewarded for its specificity and penalized for its overgeneralization can bave a strong effect on the final performance of the rule sequences discovered.

We explored the use of three different types of scoring metrics for use in selecting the "best" of the competing rules to add to the sequence. Initially we made use of a simple arithmetic difference metric, $y-s$, where $y$ (for yield) is the number of additional correct phrase labelings that would be introduced if a rule were to be added to the rule sequence, and $s$ (for sacrifice) is the number of new mistaken labelings that would be introduced by the addition of the rule. 'This is 13rill's original metric, but note that it docs not differentiate between rules whose overall improvement is identical, but whose rate of over-gencralization is not. lior cxample, a rule whose yicld is roo and sacrifice is 70 is treated as equally valuable as one whose yield is only 30 but which introduces no overgeneralization at all (sacrifice $=0$ ). This can lead to the sclection of lowprecision rules, and while small numbers of precision crrors may be patched, wholcsalc precision problems make subsequent improvement more difficult. 


\begin{tabular}{|l|ccc|ccc|}
\hline \multicolumn{1}{|c|}{ Scoring metric } & \multicolumn{3}{c|}{ Training } & \multicolumn{3}{c|}{ Test } \\
& Recall & Precision & P\&OR & Recall & Precision & PorR \\
\hline Arithmetic $(y-s)$ & 88.8 & $8 \mathbf{1} .2$ & 84.8 & 87.2 & 79.0 & 82.9 \\
Log likclihood & $8 \mathbf{1 . 9}$ & 85.7 & 78.4 & 81.0 & 73.4 & 77.0 \\
F measure, $\beta=0.8$ & 86.3 & 82.9 & 84.5 & 85.0 & $8 \mathbf{1 . 5}$ & 83.2 \\
\hline
\end{tabular}

Table 2: Comparative contributions of threc scoring measures after 100 learning cpochs.

(Training on 1495 sentences from the MUC- 6 named entitics task).

The next measure we investigated was onc advocated by Dunning (1993) which uses a log likelihood measure for estimating the significance of rare events in small populations. This measure did not improve precision or recall in the learned sequences.

The third scoring measure we investigated was the F-measure (VanRijsbergen 1979), which was introduced in information retricval to compute a weighted combination of recall and precision. The F-mcasurc is also used extensively in cvaluating information cxtraction systems at MUC (Chinchor 1995). It is defined as:

$$
F=\frac{\left(\beta^{2}+1\right) P R}{\left(\beta^{2}+P\right) R}
$$

This measure is conservative in the sense that its value is closer to precision, $P$, or recall, $\mathrm{R}$, depending on which is lower. By manipulating the $\beta$ parameter one is able to control for the relative importance of recall or precision. Preliminary exploration shows that a $\beta$ of 0.8 seems to boost precision with no significant loss in the long-term rccall or F-measurc of the rule sequences.

Table 2 summarizes the contributions of these threc error measures towards lcarning rule sequences for the MUC- 6 named entities task (for task details, see below).

\section{Evaluation}

We have applied this rule sequence approach to a variety of realistic tasks. These largely arose as part of our information extraction cfforts, and have been either directly or indirectly evaluated in the context of two evaluation conferences: MUC-6 and MLr (for Multilingual Entity 'Tagging). In this paper, we will primarily report on evaluation conducted in the context of the muc-6 named cntities task (Sundheim 1995). ${ }^{1}$

The named entities task attempts to measure the ability to identify the basic building blocks of most newswire analysis applications, e.g., named entities such as persons, organizations, and geographical locations. Also measured is the identification of some numeric cxpressions (money and percentiles), dates, and times. This task has become a classic application for finitestate pre-parsers, and indeed our work was in part motivated by the success that has been achieved by such systems in past information extraction evaluations.

We have applied a variety of techniques towards this task. The easy cases of dates and times are identified by a separate pre-processor, leaving numeric expressions

\footnotetext{
${ }^{1}$ We have also measured performance on several syntactic constructs, (e.g., the so-called noun group), and on semantic subgrammars, (e.g., person-title-organization appositions).
}

(also casy) and "proper names" (the interesting hard part) to be treated by the rule sequence processor.

\section{Hand-crafted Rules}

We first approached this task as an enginecring problem, and wrote a rule sequence by hand to identify these named entities. The rule sequence comprises 145 named-entity rules, 12 rules for expressions of money and percentiles, and 61 rules for gcographical complements (as in "Hyundai of Canada"). In addition, the rules refer to a few morphological predicates and some short word lists--onc such list, for example lists words designating business subsidiaries, e.g., "unit". "The initial phrase labeling for the proper name cases is implemented by accumulating runs of NNP- and NNPs tagged lexemes. A similar strategy is used for number expressions, using numeric tags.

The performance of our hand-crafted rule sequence is summarized in Table 3 , below, which gives component scores on the MUc- 6 blind test set. The most interesting measures are those for the difficult proper name cases. Our performance here is high, especially for person names. Our lowest score is on organizational names, but note that the system lacks any extensive organization name list. Aside from ten hard-wired names, all names are found from first principles. On the easy numeric expressions, performance is almost perfect-precision appcars poor for percentiles, but this is duc to an artifact of the testing procedure. ${ }^{2}$

\section{Machine-crafted Rules}

To evaluate the performance of our learning algorithm, we attempted to reproduce substantially the same environment as is used for the hand-crafted rules. The learner had access to the same predefined word lists, including the less-than-perfect TIPSIER gazetteer. Further, we only acquired rules for the hardest cases, namely the person, organization, and location phrases. We cut off rule acquisition after the rooth rulc.

The results for this acquired rule set are surprisingly encouraging. As Table 3 shows, these rules achieved higher recall on the very hardest phrase type (organization) than their hand-crafted counterparts, albeit at a cost in precision. Overall, however, the machine-crafted rules still lag behind. When we incorporated them into our information extraction

${ }^{2}$ Our performance vis-a-vis other Muc-6 participants placed us in the top third of participating systems. Except for the absolute highest performer, all these top-tercile systems were statistically not distinguishable from each other. 


\begin{tabular}{|c|c|c|c|c|c|}
\hline \multirow[t]{2}{*}{ Phrase type } & \multirow[t]{2}{*}{$N$} & \multicolumn{2}{|c|}{ Hand-crafted rules } & \multicolumn{2}{|c|}{ Machine-learned rules } \\
\hline & & Recall & Precision & Recall & Precision \\
\hline Organization & 49 & 85 & 87 & 87 & 79) \\
\hline Person & 348 & 94 & 94 & 78 & 79 \\
\hline Location & I09 & 94. & 87 & 82. & 68 \\
\hline Money & 74 & 99 & 97 & - -. & -.- \\
\hline Percent & 16 & 100 & 67 & $=$ & $\ldots$ \\
\hline All phrases & 2.150 & $9 \mathrm{~g}$ & $\begin{array}{r}92 \\
=91.2\end{array}$ & $\begin{array}{l}88 \\
\text { Ove }\end{array}$ & $\begin{array}{r}83 \\
=85.2\end{array}$ \\
\hline
\end{tabular}

Table 3: Performance on the muc-6 named cntitics blind test.

system, the machine-learned rules achieved an overall named entitics fi-score of 85.2 , compared to the 91.2 achieved by the hand-crafted rules. It should be noted, however, that the system loaded with these machitiecrafted rules still outperformed about a third of systems participating in the MUc- 6 cvaluation.

\section{Multilingual evaluation (MEI)}

After the Muc- 6 evaluation, the natned entity task was cxtended in various ways to make it more applicable cross-linguistically. Predictably, this was followed by a new round of evaluations: MEL. The target languages it this case were Spanish, Chinese, and Japanese. We applied our approach to all threc.

The ME'L cvaluation required actual system performance results to be kcpt strictly anonymous, which precludes our reporting here any scores as specific as we have cited for Iinglish. What we may legitimately report, however, is that we have effectively reproduced or bettered our hand-cnginecred linglish results in the Spanish and Japanese tasks, despitc havitug no native speakers of either language (and only the most rudimentary reading skills in Kanji). In both cases, we were able to exploit part-of-speccl tagging and some existing word lists for person names and locations.

lor Chinesc, although we had available a word segmenter, we had neither part-of-spech tagger, nor word lists, nor even the elementary reading skills we had for Japancse. As a result, we had to rely almost entircly on the lcarning procedure to acquire any rule sequences. Despite these impediments, we came close to reproducing our results with the English machinelcarned named entitics rule sequence.

\section{Discussion}

What is most cncouraging about this approach is how well it performs on so many dimensions. We have only reported here on name-finding tasks, but carly jnvestigations in other areas are cncouraging as well. With tule sequences that parse noun groups, for instance, we hope to reproduce the utility of other rule-sequence approaches to text chunking (Ramshaw of Marcus 1995). We are also excited by the promise of the learning procedure, not just because it learns good rules, but also because the rules it learns can be freely intermixed with hand-engincered rules. This mixedmode acquisition is unique among natural language learning procedures, and we put it to good use in building our multilingual name-tagging sequences.

Despitc results that comparc favorably to those of more mature systems, this work is still in its infancy. We still have much to explore, especially with the: kearning procedure. Indecel, while the learner induces rule sequences that perform well in the aggregate, individual rules clearly show their mechanical genesis. For instance, when the learner must break ties between identically-scoring rule candidates, it often does so in linguistically clumsy ways. At times, the learner may acquire a good contextual pattern, but may be unable to extend it to closely-related cases that would occur naturally to a linguist.

We believe these problems are solvable in the nearterm, and we have partial solutions in place already. As our techniques mature, this validates not only our particular approach to phrase-finding, but the whole ficld of language processing through rulc sequences.

\section{References}

Aberdeen, J., Burger, J., J)ay, D, Hirschman, J.., Robinson, P., of Vilain, M. 1995. "Description of the Alembic system used for Muc-6". In Pridgs. of Muc-6, Columbia Mo.

Appelt, D. H., Hobbs, J. R., Bcar, J., Isracl, D., é 'lyson, M. 1993. "liastus: A finite-state processor for information extraction from real-world text." In Predys. of rJcis-93, Chambéry, lirance.

Brill, I:. I993. A corpus-based approach to language learning. Doctoral Dissertation, Univ. of Pennsylvania.

Chinchor, N. I994. "Muc-5 evaluation metrics". In Predys. of MUC-5, Baltimore, MD.

Dunning, 'Г. 1993. "Accurate methods for the statistics of surprise and coincidence". Comput. $\operatorname{Ling} \mathbf{1 9}$.

Grishman, R. 1995. "Ihe NYu system for MuC,-6, or where's the syntax?" In Predgs. of MUc-6, Columbia M1).

Jacobs, P. S., Krupka, C., \& Rau, L. 1991. "Icxicoscmantic pattern-matching as a companion to parsing". In Predgs. of the Fourth DARP Speech and Nat. Lang. Workshop, San Mateo, ca: Morgan Kaufinan.

Ramshaw, I. Marcus, M. r995. "Text chunking using transformation-based learning". In Pregs. of $3 \mathrm{rd}$ Wkshp on Very Large Corpora, Cambridge, MA.

Sundheim, B. 1995. "Named entity task definition". In. Prodgs. of MUC-6, Columbia MD.

Van Rijsbergen, C.J. 1979. Information Retrieval. London: Buttersworth. 